INPLASY

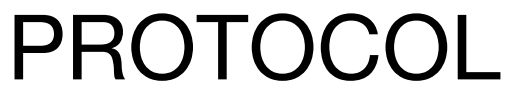

To cite: Huang et al. Efficacy and Safety of Transcranial Direct Current Stimulation on Post-Stroke Spasticity: An Updated Systematic Review with Meta-Analysis. Inplasy protocol 202130070. doi: 10.37766/inplasy2021.3.0070

Received: 19 March 2021

Published: 20 March 2021

Corresponding author: Jiapeng Huang

hjp20161107502@163.com

Author Affiliation:

Department of Rehabilitation Medicine, West China Hospital, Sichuan University

Support: National Key R\&D Plan.

Review Stage at time of this submission: Formal screening of search results against eligibility criteria.

Conflicts of interest:

None declared.

\section{Efficacy and Safety of Transcranial Direct Current Stimulation on Post-Stroke Spasticity: An Updated Systematic Review with Meta-Analysis}

Huang, JP1; Qu, Y2; Liu, LN3; Zhao, KH4; Zhao, ZQ55.

Review question / Objective: How does transcranial direct current stimulation impact spasticity in patients with stroke compared to sham transcranial direct current stimulation or other non-transcranial direct current stimulation interventions?

Condition being studied: Post-stroke spasticity.

Information sources: We searched the following electronic bibliographic databases: Cochrane Library, EMBASE, MEDLINE (via PubMed), PEDro, CBM, CNKI, and Wan Fang Data. All the English and Chinese publications until 02 March 2021 were searched without any restriction of countries or article type. The search string was built as follows: individually or combined included stroke, transcranial direct current stimulation, and a string of words that were determined after multiple pre-searches.

INPLASY registration number: This protocol was registered with the International Platform of Registered Systematic Review and Meta-Analysis Protocols (INPLASY) on 20 March 2021 and was last updated on 20 March 2021 (registration number INPLASY202130070).

\section{INTRODUCTION}

Review question / Objective: How does transcranial direct current stimulation impact spasticity in patients with stroke compared to sham transcranial direct current stimulation or other nontranscranial direct current stimulation interventions?

Condition being studied: Post-stroke spasticity. 


\section{METHODS}

Participant or population: Individuals with post-stroke spasticity. No exclusion criteria were based on the participant's condition.

Intervention: All types of transcranial direct current stimulation.

Comparator: Control interventions should use sham transcranial direct current stimulation or other non-transcranial direct current stimulaiton interventions which should be described in as much detail as the intervention.

Study designs to be included: Randomized controlled trials.

Eligibility criteria: Only randomized controlled trials published in English or Chinese, which purposed to assess the effects of transcranial direct current stimulation or transcranial direct current stimulation combined with other treatment modalities versus non-transcranial direct current stimulation treatment, such as conventional rehabilitation training, sham or routine medication, on post-stroke spasticity, will be included.

Information sources: We searched the following electronic bibliographic databases: Cochrane Library, EMBASE, MEDLINE (via PubMed), PEDro, CBM, CNKI, and Wan Fang Data. All the English and Chinese publications until 02 March 2021 were searched without any restriction of countries or article type. The search string was built as follows: individually or combined included stroke, transcranial direct current stimulation, and a string of words that were determined after multiple pre-searches.

Main outcome(s): Spasticity is obtained from the Modified Ashworth Scale measurement.

Quality assessment / Risk of bias analysis: Two reviewers will independently and separately assess the risk of bias using Cochrane Collaboration's tool. Two reviewers will independently and separately perform the GRADE (Grading of Recommendations Assessment, Development, and Evaluation) guidelines to evaluate the overall quality of the evidence.

Strategy of data synthesis: The metaanalysis will be performed using Review Manager (RevMan) software (The Cochrane Collaboration, version 5.3). For the continuous variables, the mean difference (MD) and the inverse variance method will be used to pollute the data of outcome measurements. However, standardized mean difference (SMD) will be used where different scales were used to measure the same outcome. The results of the metaanalysis will be presented using forest plots. The $\mathrm{X}^{2}$ and $\mathrm{I}^{2}$ statistics will be used for evaluating heterogeneity.

Subgroup analysis: If the necessary data are available, subgroup analyses will be done for comparing the time since stroke, age, type of transcranial direct current stimulation, stimulation intensity, duration, location of stimulation, comparison design (received sham stimulation or not), and muscle spasticity of different joints.

Sensitivity analysis: The issues suitable for sensitivity analysis are identified during the review process where the individual peculiarities of the studies under investigation are identified.

\section{Language: English or Chinese.}

Country(ies) involved: China.

Keywords: Transcranial Direct Current Stimulation; Stroke; Muscle Spasticity; systematic review; meta-analysis.

Contributions of each author:

Author 1 - Jiapeng Huang.

Email: hjp20161107502@163.com

Author 2 - Yun Qu.

Email: dr_yunqu@163.com

Author 3 - Lini Liu.

Author 4 - Kehong Zhao.

Author 5 - Ziqi Zhao. 